\title{
Influence of Ventilation Parameters on Intraabdominal Pressure
}

\author{
Claudiu Puiac ${ }^{1}$, Janos Szederjesi $^{1 *}$, Alexandra Lazar¹, Emoke Almasy¹, Paul Rad ${ }^{1}$, Lucian \\ Puscasiu² \\ ${ }^{1}$ Anesthesiology Department, University of Medicine and Pharmacy of Tirgu Mures, Romania \\ 2 Gynecology and Obstetrics Department, University of Medicine and Pharmacy of Tirgu Mures, Romania
}

\begin{abstract}
Introduction: Intraabdominal pressure monitoring is not routinely performed because the procedure assumes some invasiveness and, like other invasive procedures, it needs to have a clear indication to be performed. The causes of $\mathrm{IAH}$ are various. Mechanically ventilated patients have numerous parameters set in order to be optimally ventilated and it is important to identify the ones with the biggest interference in abdominal pressure. Although it was stated that mechanical ventilation is a potential factor of high intraabdominal pressure the set parameters which may lead to this diagnostic are not clearly named.
\end{abstract}

Objectives: To evaluate the relation between intraabdominal pressure and ventilator parameters in patients with mechanical ventilation and to determine the correlation between intraabdominal pressure and body mass index.

Material and method: This is an observational study which enrolled 16 invasive ventilated patients from which we obtained 61 record sheets. The following parameters were recorded twice daily: ventilator parameters, intraabdominal pressure, SpO2, Partial Oxygen pressure of arterial blood. We calculated the Body Mass Index (BMI) for each patient and the volume tidal/body weight ratio for every recorded data point.

Results: We observed a significant correlation between intraabdominal pressure (IAP) and the value of PEEP $(p=0.0006)$. A significant statistical correlation was noted regarding the tidal volumes used for patient ventilation. The mean tidal volume was $5.18 \mathrm{ml} / \mathrm{kg}$. Another significant correlation was noted between IAP and tidal volume per kilogram ( $p=0.0022)$. A positive correlation was found between BMI and IAP $(p=0.0049)$, and another one related to the age of the enrolled patients. $(p=0.0045)$.

Conclusions: The use of positive end-expiratory pressures and high tidal volumes during mechanical ventilation may lead to the elevation of intraabdominal pressure, a possible way of reducing this risk would be using low values of PEEP and also low volumes for the setting of ventilation parameters. There is a close positive correlation between the intraabdominal pressure levels and body mass index.

Keywords: intraabdominal pressure, mechanical ventilation, body mass index

Received: 15 December 2015 / Accepted: 14 March 2016

\section{INTRODUCTION}

In intensive care, most of patients are mechanically ventilated since respiratory failure, regardless of its cause, is the most frequent cause of Intensive Care Unit (ICU) admission. In the ICU, patients benefit from thorough invasive, as well as non-invasive monitoring.

Intraabdominal pressure monitoring is not routinely performed because the procedure assumes some inva- siveness and, like other invasive procedures, it needs to have a clear indication to be performed.

Normal intraabdominal pressure (IAP) was definedby the World Society of Abdominal Compartment Syndrome to be $10-12 \mathrm{mmHg}$, measured in the abdominal cavity. Intraabdominal hypertension (IAH) is defined above this value [1].

Intraabdominal hypertension has four degrees and is graded as follows: Grade I- IAP $=12-15 \mathrm{mmHg}$ Grade

\footnotetext{
Correspondence to: Janos Szederjesi: 38 Gheorghe Marinescu street, Tîrgu Mureș, Mureș, 540139, Romania. E-mail: yangzi37@gmail.com Puiac Claudiu: University of Medicine and Pharmacy of Tirgu Mures, 38 Gheorghe Marinescu street, Tîrgu Mureș, Mureș, 540139, Romania Lazar Alexandra: University of Medicine and Pharmacy of Tirgu Mures, 38 Gheorghe Marinescu street, Tîrgu Mureș, Mureș, 540139, Romania Almasy Emoke: University of Medicine and Pharmacy of Tirgu Mures, 38 Gheorghe Marinescu street, Tîrgu Mureș, Mureș, 540139, Romania Rad Paul: University of Medicine and Pharmacy of Tirgu Mures, 38 Gheorghe Marinescu street, Tîrgu Mureș, Mureș, 540139, Romania

Puscasiu Lucian: University of Medicine and Pharmacy of Tirgu Mures, 38 Gheorghe Marinescu street, Tîrgu Mureș, Mureș, 540139, Romania
} 
II-IAP $=16-20 \mathrm{mmHg}$ Grade III-IAP $=21-25 \mathrm{mmHg}$ and Grade IV-IAP>25 mmHg. The abdominal compartment syndrome can appear above this value, this being a potentially lethal situation [2].

The causes of IAH are various. Some of the factors that are recognized to be involved in IAH occurring in intensive care units are: sepsis, oliguria, renal failure [3], major trauma, abdominal surgery [4], acute pancreatitis, obesity, hypotension and shock [5], increased severity scores [6], massive fluid resuscitation [6].

Because the abdomen takes part in respiratory mechanics, it is to be expected that some modifications in the respiratory apparatus will echo in the abdominal cavity. Mechanically ventilated patients have numerous parameters set in order to be optimally ventilated and it is important to identify the ones with the biggest interference in abdominal pressure. Although mechanical ventilation was stated by other studies [6] as a potential factor of high intraabdominal pressure, the set parameters which may lead to this diagnostic are not clearly stated and this is why we conducted the present study. The cut off value of intraabdominal pressure is also uncertain, some authors suggests that even slightly elevated pressures may lead to increased acute systemic inflammation and acute kidney injury [7].

\section{AIMS/ OBJECTIVES}

To evaluatethe relation between intraabdominal pressure and ventilator parameters in patients with mechanical ventilation.

To determine the correlation between intraabdominal pressure and body mass index.

\section{MATERIAL AND METHODS}

This is an observational study conducted between January and April 2016 in the Intensive Care Clinic of the Emergency Clinical County Hospital of Tirgu Mures. The study enrolled 16 invasive ventilated patients, 6 were women and 10 were males, aged between 49 and 84 (mean $72 \pm 8.2$ ).

The Ethics Committee of the Hospital approved this study and patients or their legal representatives gave their written approval prior to entering the study.

The inclusion criteria were: patients ventilated invasively using oro-tracheal intubation with monitored abdominal pressure, monitored airway parameters, presence of an intra-arterial catheter.
The study protocol was as follows: the admission diagnostic and anthropometric data- weight, height, age and sex were recorded.

The following parameters were recorded twice daily, between 9 and $10 \mathrm{AM}$ and between 18 and 19 PM: ventilator parameters, using Drager Evita XL (Drager, Lubeck, Germany) ventilators intraabdominal pressure, measured with the AbViser (ConvaTec, Salt Lake City, USA) device - the measurement was done with the help of a urethro-vesical catheter, Peripheral Oxygen saturation $-\mathrm{SpO}_{2}$, measured by peripheral pulseoxymetry, Partial Oxygen pressure of arterial blood samples taken from a previously inserted arterial line. The sample was measured with a point of care type device- GemPremier4000 (Instrumentation Laboratory Company, Lexington, MA, USA).

The measurements were performed on consecutive days until the invasive monitoring was interrupted. $\mathrm{Pa}$ tients were monitored for 1 to 5 days.

The studied parameters were noted in a specially designed form: age, sex, height, weight, mode of ventilation, positive end expiratory pressure (PEEP), peak airway pressure, plateau pressure, inspiratory pressure, airway resistance, airway dynamic compliance, tidal volume, respiratory rate, oxygenation parameters$\left(\mathrm{PaO}_{2}, \mathrm{SpO}_{2}\right)$ and intraabdominal pressure.

The study included 61 data recordings from the 16 enrolled patients. We calculated the Body Mass Index (BMI) for each patient and the volume tidal/body weight ratio for every recorded data point.

The data were included in Microsoft Excel (Microsoft, Washington, USA) and statistically processed using the programs GraphPad (GraphPad Software, Inc., California, USA) and MedCalc (MedCalc Software, Ostend, Belgium).

The correlations between the different studied parameters were made using the $\mathrm{t}$-student andSpearman tests. The level of statistical significance was set for $\alpha=0.05$.

\section{RESULTS}

Analyzing the 61 datasets, we observed a significant correlation between intraabdominal pressure (IAP) and the value of PEEP $(\mathrm{p}=0.0006)$ (Table 1). Also, a very significant statistical correlation was noted regarding the tidal volumes used for patient ventilation. 
The mean tidal volume was $489 \mathrm{ml}$, which in $\mathrm{ml} /$ $\mathrm{kg}$ is $5.18 \mathrm{ml} / \mathrm{kg}$. Another significant correlation was noted between IAP and tidal volume per kilogram $(\mathrm{p}=0.0022)$.

We did not notice any statistical significant correlation between peak pressure in the airway and IAP, or with the ventilation mode.The same results were found between IAP and plateau airway pressure $(\mathrm{p}=0.4827)$ and inspiratory airway pressure $(\mathrm{p}=0.0594)$.

The ventilation mode used for the patients enrolled in the study were: continuous positive airway pressure (CPAP, $n=22,36.06 \%$ ), bi-level positive airway pressure (BIPAP, $n=47.54 \%$ ), synchronized intermittent mandatory ventilation (SIMV, $\mathrm{n}=8,13.11 \%$ ) and intermittent positive pressure ventilation (IPPV, $n=2,3.27 \%$ ). We found no statistical significant correlation between the mode of ventilation and IAP.

When looking at the airway resistance, our study failed to prove significant correlation with intra-abdominal pressure $(\mathrm{p}=0.0596)$, although the dynamic pulmonary compliance showed a significant relation with IAP $(\mathrm{p}=0.0462)$.
Regarding the level of oxygenation, we could not point out any significant correlation between IAP and any of the following parameters: $\mathrm{PaO}_{2}(\mathrm{p}=0.1188)$, $\mathrm{SpO}_{2}(\mathrm{p}=0.8707), \mathrm{FiO}_{2}(\mathrm{p}=0.9429)$.

When looking at the biometric data, a positive correlation was foundbetween BMI and IAP ( $\mathrm{p}=0.0049)$, and another one related to the age of the enrolled patients $(\mathrm{p}=0.0045)$.

Out of the studied sample, three patients had very high measurements for the intraabdominal pressure (over $20 \mathrm{mmHg}$ ), caused by surgery complications and needed surgical re-intervention.

\section{Discussions}

An abdominal pressure above the normal limit is known to be an unfavorable prognostic factor and can lead to the abdominal compartment syndrome, which can be lethal. Also, a high intraabdominal pressure can lead to organ hypoperfusion, which can end in organ failure [8].

As the actions of reducing intraabdominal pressure become more prompt, the secondary lesions which can

Table 1. Biometric and ventilation correlations with intra-abdominal pressure

\begin{tabular}{|c|c|c|c|c|c|}
\hline & Mean & Min & Max & $\begin{array}{c}\text { p value } \\
\text { (correlation with IAP) }\end{array}$ & $\begin{array}{c}\text { R value } \\
\text { (correlation coeficient) }\end{array}$ \\
\hline Age (mean, SD, years) & $72.54(8.2)$ & 49 & 84 & $0.0045^{*}$ & -0.3594 \\
\hline Gender (n) & $\begin{array}{l}F=6(37.5 \%) \\
M=10(62.5 \%)\end{array}$ & - & - & - & \\
\hline BMI (mean, SD, kg/m2) & 31.07 (3.9) & 24.49 & 37.83 & $0.0049 *$ & 0.3558 \\
\hline IAP (mean, SD, mmHg) & $12.64(10.1)$ & 5 & 51 & - & \\
\hline $\begin{array}{l}\text { Airway peak pressure } \\
\text { (mean, SD, mbar) }\end{array}$ & $16.62(6.9)$ & 14 & 33 & 0.5557 & -0.0769 \\
\hline $\begin{array}{l}\text { Airway plateau pressure } \\
\text { (mean, SD, mbar) }\end{array}$ & $15.78(4.0)$ & 10 & 25 & 0.4827 & 0.011 \\
\hline $\begin{array}{l}\text { Inspiratory pressure (mean, } \\
\text { SD, mbar) }\end{array}$ & $17.35(5.2)$ & 10 & 28 & 0.0594 & 0.2506 \\
\hline $\begin{array}{l}\text { Resistance (mean, SD, } \\
\mathrm{mbar} / \mathrm{L} / \mathrm{s} \text { ) }\end{array}$ & $28.86(50.25)$ & 11 & 203 & 0.0596 & -0.3071 \\
\hline $\begin{array}{l}\text { Compliance (mean, SD, ml/ } \\
\text { mbar) }\end{array}$ & $40.68(28.68)$ & 17.3 & 156 & $0.0462^{*}$ & -0.3439 \\
\hline PEEP (mean, SD, mbar) & $4.6(6.1)$ & 0 & 7 & $0.0006 *$ & 0.429 \\
\hline Tidal volum (mean, SD, ml) & $489(124.1)$ & 300 & 842 & $<0.0001^{*}$ & 0.5471 \\
\hline VT/weight (mean, SD, ml/kg) & $5.18(2.1)$ & 3.33 & 9.73 & $0.0022 *$ & 0.3841 \\
\hline $\mathrm{PaO} 2$ (mean, SD, mmHg) & $127.2(82.25)$ & 70 & 396 & 0.1188 & 0.2018 \\
\hline SpO2 (mean, SD, \%) & $98.15(2.3)$ & 90 & 100 & 0.8707 & 0.0212 \\
\hline FiO2 (mean, SD, \%) & $59.34(16.01)$ & 40 & 100 & 0.9429 & -0.0093 \\
\hline
\end{tabular}


be caused by a raised intraabdominal pressure have a lower occurrence, and the patient's prognostic can be improved [9].

By repeated measurements of the intraabdominal pressure, patients at risk for increased abdominal pressure- mechanically ventilated, with a high BMI, trauma patients- can be treated sooner in order to decrease intraabdominal pressure, by decompressive laparotomy, administering muscle relaxants, punctures [10-12].

Mechanically ventilated patients are exposed to the risk of an increased intraabdominal pressure, which may worsen their prognosis. Recognizing the situation as early as possible is a medical objective and for achieving it, several correlations were made by many researchers to find relationships between ventilator parameters and IAP.

Our study found positive statistically significant correlation between PEEP and IAP, results which are resembling the ones of Reintam et al. [6], although he admitted a value of PEEP above $10 \mathrm{~cm} \mathrm{H}_{2} \mathrm{O}$ as a predictive value for IAP. The mean value of PEEP in our study was $7 \mathrm{cmH}_{2} \mathrm{O}$ and,although different from the one in the literature, it is significantly correlated with the value of the intraabdominal pressure. This result points out a possible way of reducing the risk of IAH, by keeping the lowest possible values for PEEP if the patient condition allows it.

Abdominal pressure is transmitted, even in normal conditions, to the thoracic cavity and organs. Because of this the ventilation can be impairedby reduced pulmonary compliance [13]. However, in our study pulmonary compliance did not prove to be significantly related to the IAP. This was also the conclusion of Joerg Krebs [14], who stated that IAH, within some limits, does not produce significant modifications in mechanics of respiration.

The patients from our study were ventilated with a mean tidal volume of $5.18 \mathrm{ml} / \mathrm{kg}$. This small tidal volume was significantly correlated with intraabdominal pressure values, where we had a mean of $12.6 \mathrm{mmHg}$. These results complete the ones Ruiz Feron et al. [15], where in they observed that patients with IAH have an increased thoracic rigidity, so pulmonary volumes can be decreased in these patients. As a possible method of avoiding IAH we suggest using pulmonary volumes as low as $5 \mathrm{ml} / \mathrm{kg}$, volumes which proved to be correlated with the values of IAH.
The results of the study were obtained by measuring the IAP with a bladder catheter and correlating the values with the ventilator parameters. The literature states that, for a better evaluation of lung mechanics, measurement of esophageal pressure would have been useful. This way of measuring abdominal pressure will also help with ventilator parameters optimization in such patients [15].

Another parameter which we found to be positively correlated with IAP was BMI. The study performed by Reintam [6] also states that a BMI $>30$ is known to be a risk factor for IAH. Our results complied by obtaining a mean value of BMI of 31 , as a factor significantly correlated with $\mathrm{IAH}$.

\section{- CONCLUSIONS}

The use of positive end-expiratory pressures and high tidal volumes during mechanical ventilation may lead to the elevation of intraabdominal pressure, a possible way of reducing this risk would be to use low values of PEEP and also low volumes for the setting our ventilation parameters.

The pressures measured at the level of the airways as peak, plateauand inspiratory pressures proved to have no influence on the intra-abdominal pressure.Pulmonary dynamic compliancevaluesare in concordance with intraabdominal pressure values, also.

There is a close positive correlation between the intraabdominal pressure levels and body mass index.

\section{CONFLICT OF INTEREST}

The authors declare no conflict of interests regarding this study.

\section{REFERENCES}

1. Cheatham ML, Malbrain ML, Kirkpatrick A, et al. Results from the International Conference of Experts on Intra-Abdominal hypertension and Abdominal Compartment Syndrome. I. Definitions. Intensive Care Med. 2006;32:1722-32.

2. Kirkpatrick AW, Roberts DJ, De Waele J, et al. Intra-abdominal hypertension and the abdominal compartment syndrome: updated consensus definitions and clinical practice guidelines from the World Society of the Abdominal Compartment Syndrome. Intensive Care Med. 2013;39:1190-206.

3. Dalfino L, Tullo L, Donadio I, et al. Intra-abdominal hypertensionand acute renal failurein critically ill patient. 
Intensive Care Med 2008;34:707-13.

4. Balogh ZJ, Martin A, van Wessem KP et al. Mission to eliminate postinjury abdominal compartment syndrome. Arch Surg. 2011;146:938-43.

5. Reintam Blaser A, Parm P, Kitus $R$ et al. Risk factors for intraabdominal hypertension in mechanically ventilated patients. Acta Anaesthesiol Scand. 2011;55:607-14.

6. Ke L, Ni HB, Sun JK, et al. Risk factors and outcome of intraabdominal hypertension in patients with severe acute pancreatitis. World J Surg. 2012;36:171-8.

7. Smit M, Hofker HS, Leuvenink $H$, et al. A human model of intraabdominal hypertension: even slightly elevated pressures lead to increased acute systemic inflammation and signs of acute kidney injury. Crit Care. 2013;17:425.

8. Cheng, Z. Wei, X. Liu, et al. The role of intestinal mucosa injury induced by intra-abdominal hypertension in the development of abdominal compartment syndrome and multiple organ dysfunction syndrome. Crit Care. 2013:283.

9. Ke $\mathrm{L}, \mathrm{Ni} \mathrm{HB}$, Tong $\mathrm{ZH}$, et al.The importance of timing of decompression in severe acute pancreatitis combined with abdominal compartment syndrome. J Trauma Acute Care Surg.
2013;74:1060-6.

10. De Laet, E. Hoste, E. Verholen, et al. The effect of neuromuscular blockers in patients with intra-abdominal hypertension. Intensive Care Med. 2007;33:1811-4.

11. Cheatham ML, Safcsak K. Percutaneous catheter decompression in the treatment of elevated intraabdominal pressure. Chest. 2011;140:1428-35.

12. Struck MF, Reske AW, Schmidt T, et al. Respiratory functions of burn patients undergoing decompressive laparotomy due to secondary abdominal compartment syndrome. Burns. 2014;40:120-6.

13. Obeid F, Saba A, Fath J, et al. Increases in intraabdominal pressure affect pulmonary compliance. Arch Surg. 1995;130:544-7.

14. Krebs J, Pelosi P, Tsagogiorgas C, et al. Effects of positive endexpiratory pressure on respiratory function and hemodynamics in patients with acute respiratory failure with and without intraabdominal hypertension: a pilot study. Crit Care. 2009;13:R160.

15. Ferrón R, Pedregosa AT, García R, A, et al. Intraabdominal and Thoracic Pressures in Critically III Patients with Suspected Intraabdominal Hypertension. Med Intensiva. 2011;35:274-9. 